\title{
Study of the effect of oral health on physical condition of professional soccer players of the Football Club Barcelona
}

\author{
Cosme Gay-Escoda ${ }^{1}$, Dario-Miguel Vieira-Duarte-Pereira ${ }^{2}$, Jordi Ardèvol ${ }^{3}$, Ricard Pruna ${ }^{4}$, Javier Fernan- \\ dez ${ }^{5}$, Eduard Valmaseda-Castellón ${ }^{6}$
}

\begin{abstract}
${ }^{1} \mathrm{DDS}, \mathrm{MD}, \mathrm{PhD}$. Chairman and Professor of Oral and Maxillofacial Surgery. Director of the Master of Oral Surgery and Implantology. School of Dentistry of the University of Barcelona. Coordinator and researcher of Bellvitge Institute for Biomedical Research (IDIBELL). Oral and Maxillofacial Surgeon of the Teknon Medical Center, Barcelona (Spain)

${ }^{2}$ DDS. Fellow of the Master of Oral Surgery and Implantology. School of Dentistry of the University of Barcelona (Spain)

${ }^{3}$ MD. Medical Director, Fútbol Club Barcelona

${ }^{4}$ MD. Fútbol Club Barcelona

${ }^{5}$ Physiotherapist. Fútbol Club Barcelona

${ }^{6}$ DDS, PhD. Associate Professor of Oral Surgery. Professor of the Master of Oral Surgery and Implantology. School of Dentistry of the University of Barcelona (Spain). Researcher of Bellvitge Institute for Biomedical Research (IDIBELL)
\end{abstract}

Correspondence:

Centro Médico Teknon,

Instituto de Investigación IDIBELL,

Vilana 12

08022 Barcelona (Spain)

cgay@ub.edu

Received: 08/04/2010

Accepted: 30/07/2010

\author{
Gay-Escoda C, Vieira-Duarte-Pereira DM, Ardèvol J, Pruna R, Fernandez \\ J, Valmaseda-Castellón E. Study of the effect of oral health on physical \\ condition of professional soccer players of the Football Club Barcelona. \\ Med Oral Patol Oral Cir Bucal. 2011 May 1;16 (3):e436-9. \\ http://www.medicinaoral.com/medoralfree01/v16i3/medoralv16i3p436.pdf

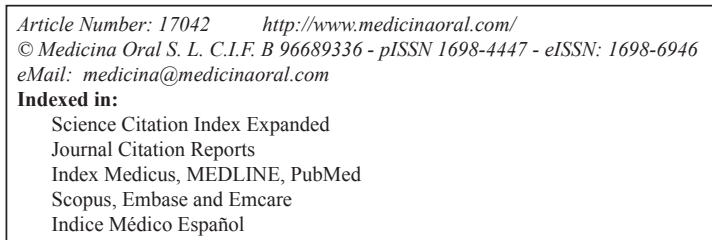

\begin{abstract}
Objectives: The objective of this study was to evaluate the oral health status of professional soccer players of F.C. Barcelona and its relation to the incidence of sport lesions.

Methods: Thirty professional soccer players were consecutively evaluated in the seasons 2003/4, 2004/5 and 2005/6. A research protocol to assess their oral health was developed. DMFT, Quigley \& Hein plaque index (PI), Löe \& Silness gingival index (GI), World Health Organization malocclusion index, Ramfjord teeth probing pocket depth (PPD), TMJ examination and history of dental trauma were recorded. All physical injuries sustained by players during the season were documented from F.C. Barcelona medical services.

Results: Mean DMFT score was 5.7 (SD 4.1), Quigley \& Hein plaque index score was 2.3 (SD 1.1), Löe \& Silness gingival index was 1.1 (SD 0.8), and periodontal pocket depth was $1.9 \mathrm{~mm}$ (SD 0.3). Pearson's analysis showed a significant correlation between PI and GI $(\mathrm{p}<0.01)$. Nine players $(30 \%)$ presented bruxism - the same proportion of those with severe malocclusion. Seven (23.3\%) players had suffered uncomplicated crown fractures. The mean incidence of physical injuries was 8 (SD 3.4) per player. PI and PPD showed a statistically significant correlation to muscle injuries $(\mathrm{p}<0.05)$.

Conclusions: Soccer players, despite intensive medical follow-up, have significant oral health problems such as untreated caries, gingivitis or malocclusion, and suffer dental trauma as a result of sports activities. Their physical condition could also be associated to oral health.
\end{abstract}

Key words: Sports injuries, caries, plaque index, gingival index, temporomandibular joint, epidemiology, dental trauma. 


\section{Introduction}

Soccer is one of the most popular team sports in the world, with approximately 200.000 professional and 240 million amateur players (1). Soccer in Spain generates over 8000 million $€$, around $1.7 \%$ of the gross national product of the country, according to a study carried out by the Spanish Professional Soccer League (Liga de Fútbol Profesional, LFP).

The high performance standards required of a soccer player can only be attained by a totally healthy individual. The time, effort and money invested in achieving this maximum level of fitness should not be put at risk by preventable general and oral health problems occurring prior to, or during competition (2).

Dentistry applied to sports focuses on the study, revision, prevention and treatment of orofacial traumatology, on athlete dental health maintenance, and on the diffusion of information and new knowledge within the sports medicine community.

A frequent concern in epidemiological studies on sport injuries is the different methodologies used, which makes interpretation and assessment difficult. Studies on the incidence of soccer injuries varies according to the definition of injury $(3,4)$. Factors that affect this incidence are the level of competition, the number of matches and the training system. Muscle injuries, torn cartilage, fractured bones, tendonitis, soft tissue lacerations, contusions, and broken teeth are just some of the injuries associated with sports activities $(5,6)$.

The frequency of soccer injuries is estimated to be approximately 1.5 to 7.6 per 1000 training hours and 12 to 35 per 1000 playing hours. The majority of injuries occur in the lower extremities, mainly in the knees and ankles $(3,6,7)$. Incidence data reflect to some extent the need for physicians, dentists, and others to take preventive therapeutical decisions and focus on the health of sportsmen.

Improvement in sports injury prevention depends of epidemiological research. Accordingly, the aim of this study was to assess oral health among professional soccer players, and to explore possible correlations between oral status and the occurrence of sport injuries.

\section{Material and Methods}

A cross-sectional and descriptive epidemiological study was carried out during the 2003/4, 2004/5 and 2005/6 seasons on a sample of 30 professional soccer players of the F.C. Barcelona. Thirty professional players belonging to the F.C. Barcelona soccer team were explored and included in the study. A standardized screening protocol was developed for assessing the oral health status of soccer players. Information on the aim of the study was supplied to the F.C. Barcelona Medical Department and to the soccer team directors, who later informed and motivated the players to participate in the study. All clinical examinations and interviews were carried out at the F.C. Barcelona Miniestadi medical infirmary, and were recorded by the same dentist in order to minimize inter-examiner variability. Oral examinations were conducted with artificial light using diagnostic dental equipment.

The decayed, missing and filled index (DMFT) was used to assess caries prevalence. The number of decayed (D), missing (M) and filled (F) teeth were recorded. To evaluate oral hygiene, a plaque index (PI) according to Quigley \& Hein was used (8). To assess periodontal status, the Löe \& Silness gingival index (GI) (9), and Ramfjord teeth probing pocket depth (PPD) in millimeters (mm) were recorded with a HuFriedy graduated periodontal probe (PCP11, HuFriedy, Chicago, USA).

The Angle molar class, the OMS malocclusion index, parafunctional habits, degree of mouth opening, palpation of masticatory muscles and presence of TMJ sounds detected with digital palpation were recorded. Sound detection was done manually because with the stethoscope it is considered unreliable (10). The players also answered a dental traumatology questionnaire. The causes and types of all injuries sustained by players during the one-year follow-up period corresponding to the seasons were documented by the team physician and collected from the F.C. Barcelona medical services. We defined injury as any physical complaint that resulted in absence from a subsequent match or training session. An injury was defined as any physical complaint caused by soccer that resulted in absence from a subsequent match or training session $(1,3)$. Physical injury types were classified according to their location and mechanism as intrinsic lesions - muscle injury, tendonitis (Achilles tendon or knee tendon), ligament injury, joint injury, bone injury - and extrinsic lesions (lacerations and contusions caused by extrinsic factors, particularly contact between players).

The data obtained were registered introduced in a database and analyzed with version 14.0 of the Statistical Package for the Social Sciences (SPSS; SPSS Inc. Chicago. USA). Descriptive and frequency distribution measures were used for the quantitative and qualitative variables, respectively. Kolmogorov-Smirnov analysis was used to test normality of distribution. Chi-square and Fisher testing was used to compare qualitative variables. Correlations between quantitative variables were measured with the Pearson's correlation coefficient. In all cases, $\mathrm{p}<0.05$ was used for the level of significance.

\section{Results}

Mean player age was 21 (SD 1.6) years. They were all active sportsmen for an average of 12.9 years (SD 2.4 years), and trained 4-5 times a week (for a total of 8-12 hours a week). Fifteen players (50\%) were regularly seen by dentists and had undergone a check-up during the last 
year, though $6(20 \%)$ had not visited a dentist for over 5 years. Eighteen $(60 \%)$ brushed their teeth two or more times a day. However, only 4 (13.4\%) used dental floss or mouthrinses. The most frequent symptom reported by players was gum bleeding in 18 players $(60 \%)$.

Mean DMFT was 5.7 (SD 4.1). Regarding the DMF components, mean active caries was 2.2 (SD 3), the filled component was 2.9 (SD 3.1), and the missing component was 0.6 (SD 1.0). The mean PI score was 2.3 (SD 1.1), with a mean GI score of 1.1 (SD 0.8), and a mean PPD score of 1.9 (SD 0.3). Pearson's analysis showed a significant $(\mathrm{p}=0.006)$ positive correlation $(\mathrm{r}=0.487)$ between PI and GI. There was no correlation between PPD and PI or GI.

The TMJ screening survey showed a mean unassisted vertical opening of $51.1 \mathrm{~mm}$ (SD 4.9), and a mean assisted opening of $54.6 \mathrm{~mm}$ (SD 4.6). Mean lateral excursion was $11.3 \mathrm{~mm}$ (SD 1.7). Two players (6.7\%) had uncorrected deviation during vertical jaw opening. Clicking was detected in 5 players $(16.7 \%)$. Palpation of the TMJ caused pain in 2 players $(6.7 \%)$. No tenderness of the masticator muscles was reported at palpation. Nine players $(30 \%)$ showed bruxism. Eighteen players $(60 \%)$ had class I (Angle) occlusion, while $6(20 \%)$ had class III occlusion, and the same percentage Class II occlusion. According to the WHO classification, severe malocclusion was present in 9 players (30\%). Eleven had no malocclusion. There was no correlation between severity of malocclusion and evidence of traumatic dental injuries $\left(\mathrm{X}^{2}=1.999 ; \mathrm{DF}=2 ; \mathrm{p}=0.368\right)$.

Five players (16.7\%) had experienced tooth pain during training or competition. Of these, 3 received no treatment, one had been prescribed medication, and only 2 were treated by the dentist. Twelve players $(40 \%)$ had suffered trauma directly to the TMJ. Seven (23.3\%) had suffered uncomplicated crown fractures of the maxillary central incisor, which was the most frequent traumatic injury. Treatment consisted of operative dentistry in 5 cases, while 2 players received no treatment. Nine $(30 \%)$ had suffered oral soft tissue laceration. The causes of soccer dental injuries were collision/contact between players in 8 cases $(61.5 \%)$, a blow from the ball in $3(23.1 \%)$, and falls in 2 cases $(15.4 \%)$. Players who had worn orthodontic appliances suffered more mouth laceration (Fisher test, $\mathrm{p}=0.021$ ). Although none of the players wore a mouth guard, 7 (23.3\%) considered such a measure to be important for preventing oral injuries in soccer.

The mean season injuries incidence per player was 8 (SD 3.4). Five (SD 2.1) were sustained in training and 2.4 (SD 1.7) during official competition matches. The mean number of intrinsic lesions was 5.5 (SD 2.6); of these, 3.2 (SD 2) were muscle injuries, 0.3 (SD 0.5) tendon injuries, 0.8 (SD 0.9) ligament injuries, 0.9 (SD 1.1) joint injuries, and 0.2 (SD 0.5) were bone injuries. The mean number of traumatic injuries due to extrinsic factors was 2.6 (SD 1.8).

PI showed a statistically significant correlation $(p=0.022$, $r=0.418$ ) to intrinsic injuries. Both PI and Ramfjord teeth PPD showed statistically significant correlations $(\mathrm{p}=0.022$ and $\mathrm{p}=0.032)(\mathrm{r}=0.416$ and $\mathrm{r}=0.392$, respectively) to muscle injuries. In the case of the DMF index and the other variables, no statistically significant correlations to physical injuries were observed.

\section{Discussion}

Many factors, including the level of competition, level of exposure, and definition of injury, have resulted in wide variations in the incidence of soccer injuries and have made comparative analysis problematic. According to our results, intrinsic injuries were more frequent than those caused by contact.

It is possible to compare the DMF index of professional football players, which are athletes with very strict health care, with dental students and medical students from the University of Barcelona, all living in the same geographical area, with a similar age and with awareness of importance of health care. The DMF index in players was 5.9, while in dental students it is 5.0 and in medical students only 3.4. Regarding the DMF components, mean active caries was considerably greater among soccer players (2.2 vs. 1.2 and 0.9 in medical and dental students, respectively). On the other hand, the filled component was clearly higher among dental students (4.1 vs. 3.1 in soccer players, and 2.0 in medical students) (11). Soccer players had more active caries then medical and dental students. This can be explained because dental students are more sensitive to the treatment of caries. In addition, a published report concluded that soccer players on an elite level may have a somewhat higher risk of developing caries than nonplayers (12).

The association between plaque and gingivitis had already been demonstrated by Löe et al. (13) The significant $(\mathrm{p}=0.006)$ positive correlation $(\mathrm{r}=0.509)$ between the Löe \& Silness gingival index and the Quigley \& Hein plaque index confirms this observation. Furthermore, epidemiological studies have demonstrated a correlation between plaque, gingivitis and periodontal tissue destruction. Soccer players showed no periodontitis, probably because of their youth.

The mean unassisted and assisted vertical opening values, as well as clicks, and the prevalence of Angle molar class I and II was similar to the mean in community controls in Spain (10). However, class III occlusion was more frequent among soccer players. Two-thirds of the players had some kind of malocclusion (post-normal occlusion, overjet exceeding $8 \mathrm{~mm}$, incompetent lips, mouth breathing). In this context, they could be more exposed to traumatic dental injury (14). 
Dental trauma suffered by $21.4 \%$ of soccer players in our study agrees with the mean $23 \%$ reported in soccer players (15). The most frequent causes of injury observed are similar to those previously reported $(14,16,17)$.

The impacted lower third molar has been considered a risk factor for mandibular angle fractures, and prophylactic removal has been recommended (18). Although the majority of soccer players in our sample had impacted third molars, they had not suffered any mandibular fracture. The very low incidence of mandibular fracture in soccer speaks against prophylactic removal.

The high performance standards required of soccer players can only be attained by totally healthy individuals. Excellent dental attention to high level soccer players is a complicated task, due to numerous competitions that do not allow adequate planning of medical decisions.

Oral examinations before the beginning of the soccer season, and periodical follow-ups by dentists, are important to prevent the occurrence of caries, periodontal disease and other oral pathologies, and to prevent dental trauma. Up to now, this is not the case even in high-level professional soccer teams.

Continued use of a standardized screening protocol could allow state-specific data to be made available on the oral health status of this population; trends could be tracked, and needs could be identified, with the development of strategies to meet such needs.

\section{References}

References with links to Crossref - DOI

1. Junge A, Rösch D, Peterson L, Graf-Baumann T, Dvorak J. Prevention of soccer injuries: a prospective intervention study in youth amateur players. Am J Sports Med. 2002;30:652-9.

2. Soler Badia D, Batchelor PA, Sheiham A. The prevalence of oral health problems in participants of the 1992 Olympic Games in Barcelona. Int Dent J. 1994;44:44-8.

3. Hägglund M, Waldén M, Ekstrand J. Exposure and injury risk in Swedish elite football: a comparison between seasons 1982 and 2001. Scand J Med Sci Sports. 2003;13:364-70.

4. Junge A, Dvorak J. Influence of definition and data collection on the incidence of injuries in football. Am J Sports Med. 2000;28:S40-6.

5. Pinkham JR, Kohn DW. Epidemiology and prediction of sportsrelated traumatic injuries. Dent Clin North Am. 1991;35:609-26.

6. Junge A, Dvorak J, Graf-Baumann T. Football injuries during the World Cup 2002. Am J Sports Med. 2004;32:23S-7S.

7. Dvorak J, Junge A. Football injuries and physical symptoms. A review of the literature. Am J Sports Med. 2000;28:S3-9.

8. Quigley GA, Hein JW. Comparative cleansing efficiency of manual and power brushing. J Am Dent Assoc. 1962;65:26-9.

9. Löe H. The Gingival Index, the Plaque Index and the Retention Index Systems. J Periodontol. 1967;38:610-6.

10. Dworkin SF, Huggins KH, LeResche L, Von Korff M, Howard J, Truelove E, et al. Epidemiology of signs and symptoms in temporomandibular disorders: clinical signs in cases and controls. J Am Dent Assoc. 1990;120:273-81.

11. Cortes FJ, Nevot C, Ramon JM, Cuenca E. The evolution of dental health in dental students at the University of Barcelona. J Dent Educ. 2002;66:1203-8.

12. Ljungberg G, Birkhed D. Dental caries in players belonging to a Swedish soccer team. Swed Dent J. 1990;14:261-6.

13. Loe H, Theilade E, Jensen SB. Experimental gingivitis in man. J Periodontol. 1965;36:177-87.

14. Forsberg CM, Tedestam G. Etiological and predisposing fac- tors related to traumatic injuries to permanent teeth. Swed Dent J. 1993;17:183-90.

15. Ferrari CH, Ferreria de Mederios JM. Dental trauma and level of information: mouthguard use in different contact sports. Dent Traumatol. 2002;18:144-7.

16. Sane J, Ylipaavalniemi P. Dental trauma in contact team sports. Endod Dent Traumatol. 1988;4:164-9.

17. Kvittem B, Hardie NA, Roettger M, Conry J. Incidence of orofacial injuries in high school sports. J Public Health Dent. 1998;58:28893.

18. Yamada T, Sawaki Y, Tohnai I, Takeuchi M, Ueda M. A study of sports-related mandibular angle fracture: relation to the position of the third molars. Scand J Med Sci Sports. 1998;8:116-9.

\section{Acknowledgements:}

This study was conducted by the oral and maxillofacial pathology and treatment research group of the Bellvitge Institute for Biomedical Research (IDIBELL Institute), with financial support from the Master of Oral Surgery and Implantology program-healthcare agreement to the University of Barcelona, the Consorci Sanitari Integral and the Servei Català de la Salut of the Generalitat de Catalunya, Barcelona, Spain. 